\title{
Credible Fuzzy Classification based Technique on Self Organized Features Maps and FRANT IC-RL
}

\author{
Mona Gamal * \\ Mansoura University, \\ Faculty of Computer and \\ Information Sciences \\ Information System DepartmentP \\ P.O.Box: 35516
}

\author{
Elsayed Radwan ${ }^{2}$ \\ ${ }^{2}$ Egypt, Mansoura University, \\ Faculty of Computer and \\ Information Sciences \\ Computer Science Department, \\ P.O.Box: 35516 \\ ${ }^{3}$ Deanship of Scientific Research, \\ Umm Al-Qura university, KSA
}

\author{
Adel M.A. Assiri ${ }^{3}$ \\ ${ }^{3}$ Biochemistry Department, \\ Faculty of Medicine, Umm Al-Qura \\ University, KSA
}

\begin{abstract}
Handling uncertainty and vagueness in real world becomes a necessity for developing intelligent and efficient systems. Based on the credibility theory, a fuzzy clustering approach that improves the classification accuracy is targeted by this work. This paper introduces a design of an efficient set of fuzzy rules that are inferred by a hybrid model of SOFM (Self Organized Features Maps) and FRANTIC-SRL (Fuzzy Rules from ANT-Inspired Computation - Simultaneous Rule Learning). Self-Organized Features Maps cluster inputs using self-adaption techniques. They are useful in generating fuzzy membership functions for the subsets of the fuzzy variables. The generated fuzzy variables are ranked by means of the credibility measure wherever the weighted average of their confidence level is determined. FRANT IC-SRL builds the fuzzy classification rule set using the ranked credibility variables in a simultaneous process. Moreover, the whole fuzzy system is evaluated based on the credibility value. The details and limitations of the proposed model are illustrated. Also, the experimental results and a comparison with previous techniques in generating fuzzy classification rules from medical data sets are declared.
\end{abstract}

Keywords-Fuzzy Rule; Classification; Self-Organized Feature Map; Credibility Measure; Ant Colony Optimization

\section{INTRODUCTION}

In the learning process, uncertainty, data labeling and vagueness struggle the development of any real system. Thus, there is a need for developing intelligent and efficient systems that handle these problems. Fuzzy system[7] depends on fuzzy input-output variables instead of crisp ones. Unlike crisp variables, the values of the fuzzy variables belong to fuzzy subsets with a degree of membership. The parameters involved in such kind of programming problem are fuzzy variables, and the resulting problem is called a fuzzy programming problem. One kind of these fuzzy programming problems is the fuzzy rule based system that determines its decision through a set of fuzzy rules and some inference mechanism (inference engine). The fuzzy programming problem has been widely studied in a variety of fields, which are all based on the fuzzy set theory [7] and the concept of possibility measure [14]. Over the last decades, Fuzzy (ifthen) rules were usually derived from human experts who are affected by the perspective of the expert. Hence, many approaches were proposed to automatically generate fuzzy (if- then) rules from the training data. These approaches are always complex optimization problems with complicated feasible set.

In a hybrid intelligent approach based on fuzzy simulation, UrszulaMarkowska-Kaczmar and WojciechTrelak used genetic algorithms in optimizing Artificial Neural Networks (ANN) for extracting fuzzy classification rules[18]. However, since the ANN suffers from the problems of proneness to over fitting, and the empirical nature of the model development, the computation cost of these hybrid intelligent algorithms is time-consuming. Bilal Alatas and Erhan Akin proposed Ant Colony Optimization to induce fuzzy classification rules through different Ant Miner algorithms like FCACO [2]. FRANT IC-SRL (Fuzzy Rules from ANT-Inspired Computation - Simultaneous Rule Learning) and FRANT ICIRL(Fuzzy Rules from ANT-Inspired Computation - Iterative Rule Learning) are two different fuzzy classification rules induction algorithms for simultaneous and iterative techniques respectively[12][20]. But these researches assume that fuzzy variables are prepared or use discrimination techniques to make the membership functions of the subsets of the variables. Gene Expression Programming method uses two populations. One for Fuzzy Classification Rules and the other for membership function [1][17]. Although this research cared about the good preparing of the fuzzy variables but it ignored the credibility ranking problem that measures the confidence level (calculate the weighted average of the fuzzy variables, credibility value) of the fuzzy attributes. Xiaxia Huang introduced a study of the capital budgeting problem to calculate the credibility measure of the capital budgeting with fuzzy investment outlays and fuzzy annual net cash flows[22]. RituparnaChutia, SupahiMahanta and D. Datta were interested in how the credibility distribution of triangular fuzzy variable leads to find a different technique for generating the triangular membership function for fuzzy variables[16]. The Wilcoxon signed rank test tries to determine whether the median of a population is a specified constant by treating the observations as imprecise values. The test procedure is developed by using the concepts of Credibility Theory for studying the behavior of fuzzy phenomena[19]. Thus, a hybrid intelligent algorithm should be argued and applied to the fuzzy rule based problem to reduce the computation cost and improve the computation 
accuracy. By this paper the fuzzy rule based problem is provided under the credibility theory, which involves a weighted average based on the expected value of the fuzzy variables.

This paper generates the fuzzy rules in four stages. The first stage prepares the fuzzy variables by generating the membership function for each fuzzy subset of the variables. Self Organized Feature Maps (SOFM) [4][15], unsupervised technique, generate the membership function for each variable depending on its self-adaptation concept. Hence, a cluster based technique is first determined. Because of the deficiencies of the membership function in fuzzy mathematics that lacking of self-dual, the credibility theory is chosen to overcome this problem. The second stage ranks the generated fuzzy variables according to the credibility inversion theorem[8]. The credibility values verified that the generated fuzzy variables meet the desired confidence level. Although SOFM based on the credibility measure can map efficient analogue membership function, it still suffers from irrelevant features. These problems can be solved well by evolving the ranked fuzzy variable subsets in the learning process in purpose of reducing the feature vector and discovering the most significant fuzzy rules. In the third stage, the result of ranked fuzzy variables and the training data are passed simultaneously to the FRANT IC-SRL (Fuzzy Rules from ANT-Inspired Computation - Simultaneous Rule Learning)[12] which randomly generate initial node graph of conditional terms. The initial population is evolved by Ant Colony optimization algorithm [3][17][20] to find the best fuzzy rule base with respect to the training data. Finally, the accuracy of the generated fuzzy rules is determined. The output of the system (classes attribute values and membership degrees) is evaluated in terms of credibility measure to calculate the confidentiality degree of the whole integrated system. The details and the limitation of the proposed integrated model is illustrated by this paper. Also, the experimental results are declared on a (medical) dataset taken from UCI machine learning repository [6]. The comparison with previous techniques in generating fuzzy classification rules illustrates the efficiency of the new hybrid model.

The rest of this paper is organized as follows: Section 2 represents the preliminaries such as the declaration of the fuzzy systems, credibility theory, Self Organized Feature Maps SOFM and Ant Colony Optimization (ACO). Section 3 gives an over view on the whole system and its modules. It goes inside the system to explain in detail the generation of the fuzzy membership functions of the subsets of the fuzzy variables, ranking the generated fuzzy variables using credibility measure, designing the fuzzy rule set using the FRANT IC-SRL(Fuzzy from ANT-Inspired Computation Simultaneous Rule Learning). Then the credibility value of the whole system is calculated. Experimental results and conclusion will appear in sections 4 and 5 respectively.

\section{PRELIMINARIES}

\section{A. Fuzzy System}

Fuzzy System [7]tends to simulate human thinking in dealing with variables values( labels, words and linguistic terms). It depends on measuring vague and ambiguous terms (parameters and variables) instead of ordinary variables that have exact values. Unlike two-valued Boolean logic, fuzzy logic is multi-valued.Fuzzy variables (credibility variables) have fuzzy values which partially belong to a set of fuzzy subsets. The degree by which an element belongs to a fuzzy subset is called the fuzzy membership degree or credibility value. This degree of membership is characterized by a fuzzy membership function.

$$
\mu_{A}(u): U \rightarrow[0,1]
$$

where $U$ is called the universe of discourse and $A$ is a fuzzy subset of $U$.

Zadeh-Mamdani's fuzzy rules[7] are (if -then) rules that its conditions and decisions both consists of fuzzy variables that belongs to some fuzzy sets with some degree of membership.

\section{IF $\mathrm{x}$ is $\mathrm{A}$, THEN $\mathrm{y}$ is $\mathrm{B}$}

Where ( $\mathrm{x}$ is $\mathrm{A}$ ) and ( $\mathrm{y}$ is $\mathrm{B}$ ) are two fuzzy propositions; $\mathrm{x}$ and $y$ are fuzzy variables defined over universes of discourse $\mathrm{U}$; and $\mathrm{A}$ and $\mathrm{B}$ are fuzzy sets defined by their fuzzy membership functions

$$
\mu_{A}(u): U \rightarrow[0,1] \text { And } \mu_{B}(u): U \rightarrow[0,1]
$$

Fuzzy inference is an inference method that uses fuzzy implication relations, fuzzy composition operators, and an operator to link the fuzzy rules. Different reasoning strategies over fuzzy rules are possible. Most of them use the generalized modus ponens rule[7]. The generalized modus ponens inference law applied over a simple fuzzy rule can be expressed as follows: (IF $x$ is A, THEN $y$ is $B$ ) and ( $x$ is $A^{\prime}$ ), then ( $y$ is $B^{\prime}$ ) should be inferred. The compositional rule of inference is one way to implement the generalized modus ponens law:

$$
B^{\prime}=A^{\prime} \circ A \rightarrow B=A^{\prime} \circ R_{a b}
$$

Where:

- $\circ$ Is a compositional operator.

- $\mathrm{R}_{\mathrm{ab}}$ is a fuzzy relational matrix representing the implication relation between the fuzzy concepts A and B.

$$
R_{a b}=\operatorname{Max}_{x \in A \& y \in B}\left\{\operatorname{Min}\left(\mu_{A} x, \mu_{B} y\right)\right\}
$$

A fuzzy inference method combines the results $\mathrm{Bi}$ for the output fuzzy variable y inferred by all the fuzzy rules for a given set of input facts. In a fuzzy production system, which performs cycles of inference, all the fuzzy rules are fired at every cycle and they all contribute to the final result. Some of the main else-links between fuzzy rules are OR-link (max operator) \& AND-link(min operator). Defuzzification is the process of calculating a single-output numerical value for a fuzzy output variable on the basis of the inferred resulting membership function for this variable. Two methods for defuzzification are the center-of-gravity method (COG) and the mean-of-maxima method (MOM).

\section{B. Credibility Theory}

Credibility measures the degree of confidence given to a specific data set. Credibility theory aims at efficiently 
combine information from diverse sources: past and current data, individual risk and collective risk data, etc[11]. Credibility theory is used to calculate the confidence of the claims experience of an individual contract and the experience for the whole portfolio, to give a good approximation of the future risk resulting from holding that contract.

The general credibility formula in the linear form

$$
\mathrm{Cr}=\mathrm{z} \mathrm{R}+(1-\mathrm{z}) \mathrm{H}, \quad 0 \leq \mathrm{z} \leq 1
$$

where $\mathrm{z}$ is the accepted level of credibility, $\mathrm{R}$ the data event of the information credibility which is calculated for, (1$\mathrm{z}$ ) represents the complement credibility, $\mathrm{H}$ is the complement of the data event $\mathrm{R}$.

Let $£$ be a nonempty set, and let $\mathrm{P}$ be the power set of $£$ (i.e., all subsets of $£$ ). Each element in $\mathrm{P}$ is called an event. The set function $\mathrm{Cr}$ on the power set $\mathrm{P}$ is called a credibility measure if it satisfies[10]

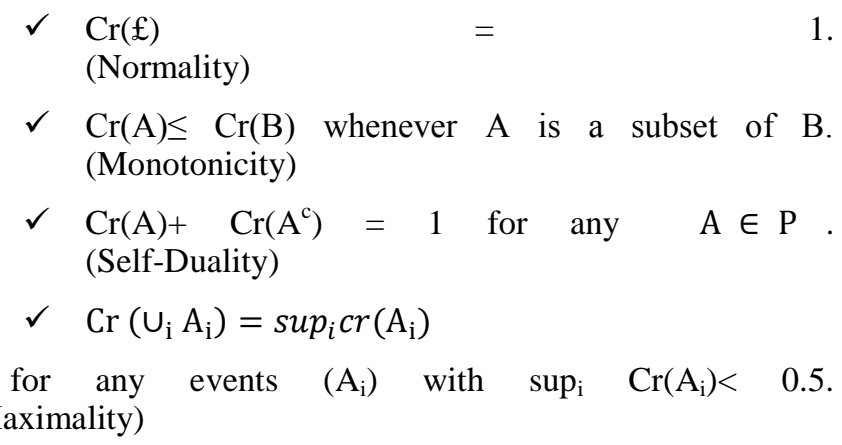

Fuzzy thinking is a way of expressing variables values in the form of words and labels like tall, very tall, short and etc. Credibility theory, in first place, was thought of as a field of mathematics for studying the behavior of fuzzy phenomena. An alternate version of credibility theory to handle the fuzzy variables environment was defined in. This definition is called credibility theory in a fuzzy environment (CT-F)[8][9][11]. CT-F involves a weighted average of the fuzzy variables confidence level based on the concepts of possibility and necessity measures.

According to the Product Credibility Theorem[10], there are some definitions that link the fuzzy variable with its credibility measure.

- A fuzzy variable is a function from a credibility space ( $\xi ; \mathrm{P} ; \mathrm{Cr}$ ) to the set of real numbers.

Let $\xi$ be a fuzzy variable defined on the credibility space $(\xi ; \mathrm{P} ; \mathrm{Cr}$ ). Then its membership function is derived from the credibility measure by

$$
\mu(x)=(2 \operatorname{Cr}\{\xi=x\}) \wedge 1, \quad x \in R .
$$

The previous definitions help to get the membership function of the fuzzy variables from its credibility measure.

\section{Credibility Extension Theorem}

Credibility Extension Theorem[21] supposes that $\theta$ is a nonempty set and $\operatorname{Cr}\{\theta\}$ is a nonnegative function on $\theta$ satisfying the credibility extension condition

$$
\begin{gathered}
\sup _{\theta \in \Theta} \operatorname{Cr}\{\theta\} \geq 0.5, \\
\operatorname{Cr}\left\{\theta^{*}\right\}+\sup _{\theta \neq \theta^{*}} \operatorname{Cr}\{\theta\}=1 \text { if } \operatorname{Cr}\left\{\theta^{*}\right\} \geq 0.5
\end{gathered}
$$

Then $\operatorname{Cr}\{\theta\}$ has a unique extension to a credibility measure on $P(\theta)$ as follows

$\operatorname{Cr}\{A\}=\left\{\begin{array}{c}\sup _{\theta \in A} \operatorname{Cr}\{\theta\}, \quad \text { if } \sup _{\theta \in A} \operatorname{Cr}\{\theta\}<0.5 \\ 1-\sup _{\theta \in A^{c}} \operatorname{Cr}\{\theta\}, \quad \text { if } \sup _{\theta \in A} \operatorname{Cr}\{\theta\} \geq 0.5\end{array}\right.$

Credibility extension theory defines the credibility measure in numerical form basing on credibility value of each singleton set. Because of the impossibility to measure the credibility value for all events, the nontrivial value for the credibility measure cannot be calculated. The credibility extension theory determines a sufficient condition for the credibility measure.

\section{Credibility Inversion Theorem}

Liu and Liu defined the credibility in a fuzzy environment as the average of the possibility and necessity measure [10]. For a fuzzy variable $\xi$ with membership function $\mu_{\xi}(\mathrm{x})$ and for any set $\mathrm{A}$ of real numbers, credibility measure of fuzzy event $\{\xi \in A\}$ is defined as

$$
\operatorname{Cr}\{\xi \in A\}=\frac{1}{2}(\operatorname{Pos}\{\xi \in A\}+\operatorname{Nec}\{\xi \in A\})
$$

for any event $\mathrm{A}$

$$
\begin{aligned}
& \operatorname{Pos}\{\xi \in A\}=\sup _{x \in A} \mu_{\xi}(x) \\
& \operatorname{Nec}\{\xi \in A\}=1-\sup _{x \in A^{c}} \mu_{\xi}(x)
\end{aligned}
$$

Using the credibility inversion theorem, the rank rate of credibility can be calculated basing on making some assumption that the fuzzy variable belong to event $A$ and calculating the average of the supreme of the membership functions for that assumption and the complement of it. Credibility Inversion Theorem uses the membership function definition of the fuzzy variable to calculate its credibility value.

\section{Self Organized Feature Map}

The Self-Organized Feature Maps (SOFM)[4][15] is an unsupervised neural network that is capable of learning its weights from its input vector without any additional information such as the corresponding output vector. SOFM is usually a two-layered network where the neurons in the output layer are organized into either a one or two-dimensional lattice structure. The SOFM solves difficult high-dimensional and nonlinear problems such as feature extraction and classification of images and acoustic patterns, adaptive control of robots, and equalization, demodulation, and error-tolerant transmission of signals in telecommunications [15].

As illustrated in Figure 1, a simple structure for the SOFM is representedwhere the d-vector is the vector of input neurons in the input layer for the following input data vector $x_{n}=\left[x_{n 1}\right.$ $\left.\mathrm{x}_{\mathrm{n} 2} \ldots \mathrm{x}_{\mathrm{nd}}\right]^{\mathrm{T}}$ and The synaptic weight vector at neuron $\mathrm{j}$ in the output layer is denoted by $\mathrm{w}_{\mathrm{j}}=\left[\mathrm{w}_{\mathrm{j} 1} \mathrm{w}_{\mathrm{j} 2} \ldots \mathrm{w}_{\mathrm{jd}}\right]^{\mathrm{T}}, \mathrm{j}=1,2, \ldots, \mathrm{J}$, where $\mathbf{J}$ is the total number of neurons in the output layer and $\mathrm{w}_{\mathrm{jk}}, \mathrm{k}=1,2, \ldots, \mathrm{d}$, is the connecting weight from the $\mathrm{j}^{\text {th }}$ neuron in the output layer to the $\mathrm{k}^{\text {th }}$ neuron in the input layer. 


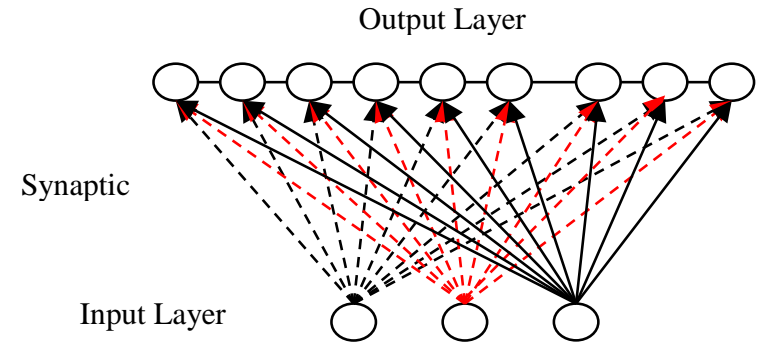

Fig. 1. a simple structure for the SOFM

\section{Ant Colony Optimization}

Ant algorithms are heuristic search mechanisms for searching and optimizing solutions. The algorithms are inspired by the various behaviors of the ants' communications. These communications is enabled by causing changes to the environment. These changes are the pheromone-laying behavior of the ants. Ant Colony Optimization (ACO)[3][17][20] is a probabilistic technique that simulates real ants for solving artificial intelligence problems which can be formulated as finding optimal paths (solutions) between nest and food places.

Ant Colony Optimization (ACO) [3][17][20] algorithms are efficiently implemented in various classification problems. There are numerous algorithms for ACO in the field of designing crisp or fuzzy classification rules. cAnt-Miner (Continuous Ant-Miner) [5], an implementation of an ACO algorithm for the classification problems using continuous attributes of data mining, are able to compete with reliable classification-rule designing algorithms. FC-ACO (Fuzzy Classification Ant Colony Optimization) [2] is a fuzzy classification rule mining algorithm based on the ACO. FRANT IC-SRL (Fuzzy Rules from ANT-Inspired Computation - Simultaneous Rule Learning) and FRANT ICIRL (Fuzzy Rules from ANT-Inspired Computation Iterative Rule Learning) are two different fuzzy classification rules induction algorithms for simultaneous and iterative techniques respectively[12]. The fundamentals of ACO are appropriate problem representation (node graph), probabilistic transition rule, local heuristic value, fitness function determination, pheromone update rule and the constraint satisfaction method. Any algorithm that defined on ACO should satisfy these fundamentals optimally.

\section{SElF ORGanized FeATURE MAP AND FRANT IC-SRL IN DESIGNING FUZZY RULES}

The target of this paper is to suggest a novel clustering approach to improve the clustering task for uncertain knowledge with labeling data values. Clustering based on fuzzy relationship is usually more flexible and dynamic. Such a kind of fuzzy relationship is the fuzzy if-then rule. The fuzzy rules are simple if-then rules but with fuzzy variables. This paper builds these fuzzy rules in four phases wherever the shortcoming in any phase is covered by the other. The first phase generates the membership function for the subsets of the fuzzy variables. Thus, a cluster based technique is first recognized. These fuzzy clusters suffer from the lacking of self-dual problem. The second phase uses the credibility inversion theorem to calculate the credibility value of the generated fuzzy variables to rank only the most credible attributes. Since the superfluous attribute, dispensable fuzzy variable, causes the redundancy problem and degrades the classification robustness, the adaptation of two corresponding fuzzy rule trails during algorithm execution that take into account the cumulated search experience, construction heuristics, is needed. The third phase designs the fuzzy rule using a set of training data and checks for its applicability on the test data. Finally, the fourth phase evaluates the credibility value of the whole system using the classes attributes resulted from the system. Hence, based on the credibility measure, Self Organized Feature Map, FRANT IC-SRL(Fuzzy Rules from ANT-Inspired Computation - Simultaneous Rule Learning) are integrted for generating the membership functions[4] and designing the fuzzy rules respectively. The credibility measure is used to rank the fuzzy variables (phase two) and calculate confidence level of the whole system (phase four). The framework of the hybrid model that outlines the main modules is illustrated in Figure 2. 


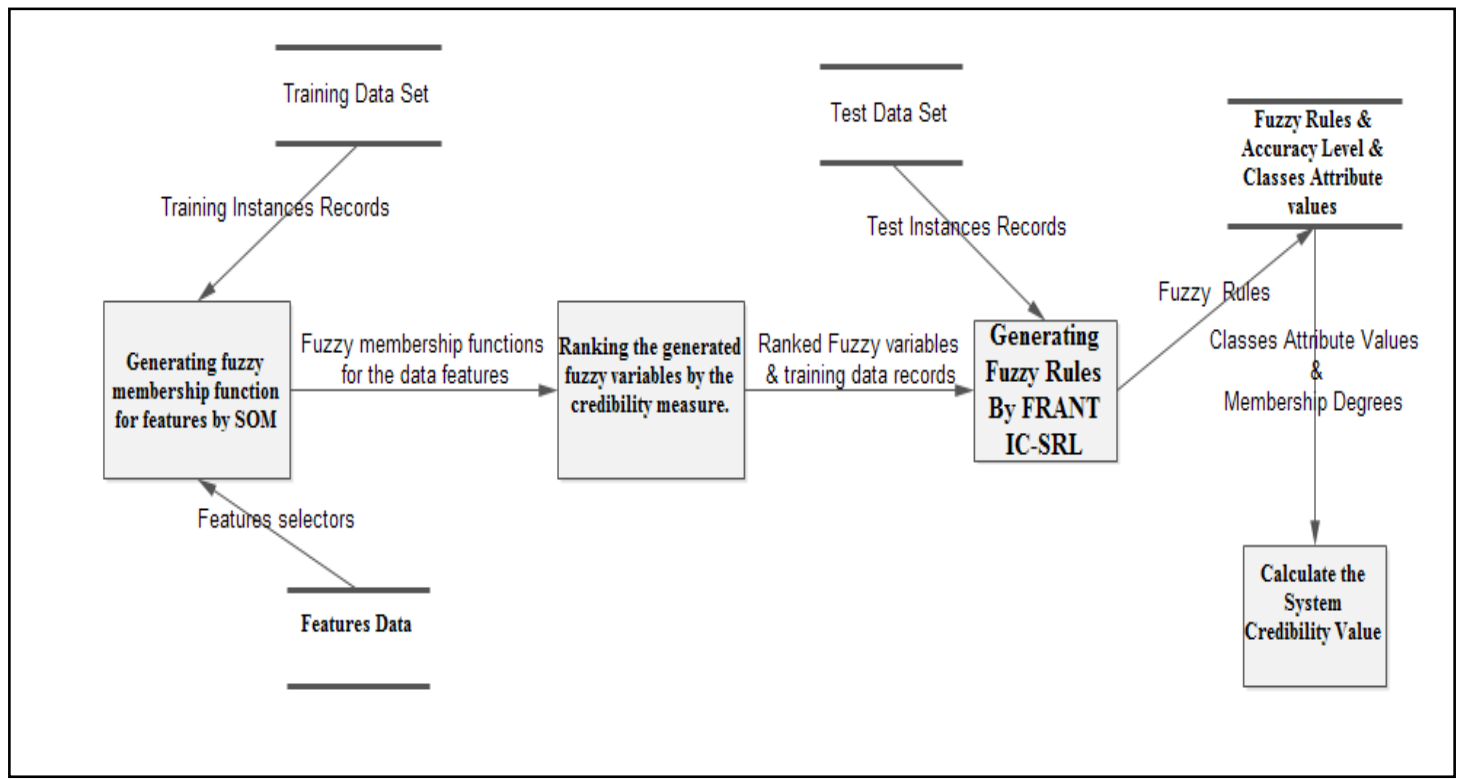

Fig. 2. The framework of the hybrid model between the SOM \& FRANT IC_SRL to design fuzzy rules Based on credibility Measure

The main components are summarized as follows:

Generating fuzzy membership function for features subsets: the SOFM capabilities of unsupervised learning and clustering generate the membership functions of the features subsets.

Ranking the fuzzy variables using the credibility measure: the weighted average of the confidence level for the generated fuzzy variables is measured by the credibility inversion theorem.

Generating fuzzy rules: FRANT IC-SRL (Fuzzy Rules from ANT-Inspired Computation - Simultaneous Rule Learning) is used as a simultaneous technique for finding the best fuzzy rule base using the Ant Colony Optimization algorithm.

Calculating credibility measure for the whole system: the credibility value of the whole system is determined through the classes attributes resulted from testing the system on the unseen instances.

\section{A. Generating Membership Functions Using Self Organized Feature Map}

The process of generating membership functions is divided into two phases. The first phase generates the proper clusters of the feature data. The other phase, fuzzy membership function is generated in correspondence with these clusters. The membership functions are generated in one phase by combining the variable labels with the variable values in the input layer of the SOFM[4]. This technique in generating fuzzy membership function with Self-Organizing Feature Map is first introduced by Chih-Chung Yang, N.K. Bose[4],[13].
In the learning process, the input vector $X_{n}=$ $\left(v, S_{1}, S_{2} \ldots S_{d}\right)$ where $v$ is the value of the feature. It should be measured to find the membership function of its fuzzy subsets $S_{1}, S_{2} \ldots S_{d}$ that discern the corresponding fuzzy values. These input vectors will be the training dataset and $v$ is handled by the expert through Nsubset.

The SOFM determine its architecture through the learning phase and update its weights according to the learning procedure. The input layer of the SOFM is composed of one neuron for each fuzzy variable value and $\mathrm{N}$ neurons for $\mathrm{N}$ subsets of the fuzzy variable. The algorithm of the SOFM is declared in Figure 3.a and 3.b.

The algorithm which is illustrated by Figure 3 go through four main steps 1) initialization for the weights randomly, 2) a competitive step where compute its value from a discrimination function as represented from step 4 till step 8 in Figure 3.a.

The particular neuronwith the minimum value of the discriminant function is chosen as the winner. 3) Cooperative step where the winning neuron determines its closest neighbors of excited neurons. Hence, cooperation among neurons is determined, as illustrated in Figure 3.b. 4). Finally, adaptation method where an adjustment for the connection weighs is measured. The weight vector of the winning neuron $w_{i}$ is updated and its neighbor neurons toward the input vector $x$, as declared in Figure 3.b step 2. Repeated presentations of thetraining data thus leads to topological ordering. 
Input: input_neurons, output_neurons, Training_Data

set, Test_Dataset.

Output: fuzzy variable membership function.

// Learning_phase

1: Initialize_SOFM (input_neurons, output_neurons)

2: Randomly_Initialize_SOFM_Weights ()

3: While Error>threshold do

4: foreach Record in Training_Data

5: Input_Record ();

6: Winning_Neuronq $\mathrm{j}_{\mathrm{j}}=$

$$
q\left(x_{n}\right)=\min _{\forall j}\left\|x_{n}-w_{j}\right\|
$$

7: Update_weights (Winning_Neuronq ${ }_{\mathrm{j}}$ );

8: end foreach;

9: Error= Calculate_ErrorRate ();

\section{0: end while}

11: Retrieving_phase (Test_Dataset);

12: Output_Memebership_Function

(Network_Weights)

Fig. 3. a: generating membership function by SOFM Algorithm

$$
\begin{aligned}
& \text { Update_weights } \\
& \text { Input: Winning_Neuronq } q_{j} \\
& \text { Output: Updated_Weights } \\
& \text { 1: Find (Winning_Neuronq } \mathrm{j}_{\mathrm{j}} \text { ) } \\
& \text { 2: } \eta_{q j}[t]= \begin{cases}\mu[t] & j \in N_{q} \\
0 & j \notin N_{q}\end{cases} \\
& \text { 2: } w_{j}[t+1]=w_{j}[t]+\eta_{q j}[t]\left(x_{n}[t]-w_{j}[t]\right) \\
& \text { 3: Output (updated_Weights }
\end{aligned}
$$

Fig. 3. b: update weights process in SOFM Algorithm

\section{B. Ranking the generated fuzzy variables using the credibility measure.}

The generated membership functions of the fuzzy variables, resulted from the SOFM, need to be evaluated to get confident about their ability to represent the data features efficiently. This evaluation procedure helps in giving the credibility to the whole system. The credibility theory aims to evaluate the weighted average of the confidence level given to a contract or a feature basing on the experience of its historical portfolio. Using the credibility inversion theorem[10], the rate of credibility can be calculated based on making some assumption that the fuzzy variable belong to an event $A$ and calculating the average of the possibility that the event $A$ happens and the necessity of the event complement is also happen. The credibility measureis given by equation (13):

$$
\operatorname{Cr}\{\xi \in A\}=\frac{1}{2}\left(\sup _{x \in A} \mu_{\xi}(x)+1-\sup _{x \in A^{c}} \mu_{\xi}(x)\right)
$$

The procedure of ranking fuzzy variables takes each fuzzy variable $\xi$ at time and randomly selects a fuzzy number $r$. The fuzzy number is used to make the event assumption $A$ as $\xi \in$ subsetofr. The procedure generates a sufficiently large number of fuzzy numbers that belong to the event $A$ and calculates the membership degrees of these numbers in the event $A$ and its complement $A^{c}$,i.e $\xi \notin$ subsetofr. The procedure puts the membership degrees in two separate groups ( $A$ membership or $A^{\mathrm{c}}$ membership). The average of the supreme function of the membership degrees of the two groups is calculated which is denoted as the credibility value. The flowchart of ranking fuzzy variables procedure is illustrated in Figure 4. After ranking fuzzy variables the process produces the credibility value, the most credible attributes are chosen as the ones with credibility value exceeding the predefined accepted confidence rate $\alpha$. Figure 5 illustrates an example forrepresenting of the $M C V$ data liver feature[6] as a fuzzy variable (generated by SOFM) with a random number $r$.

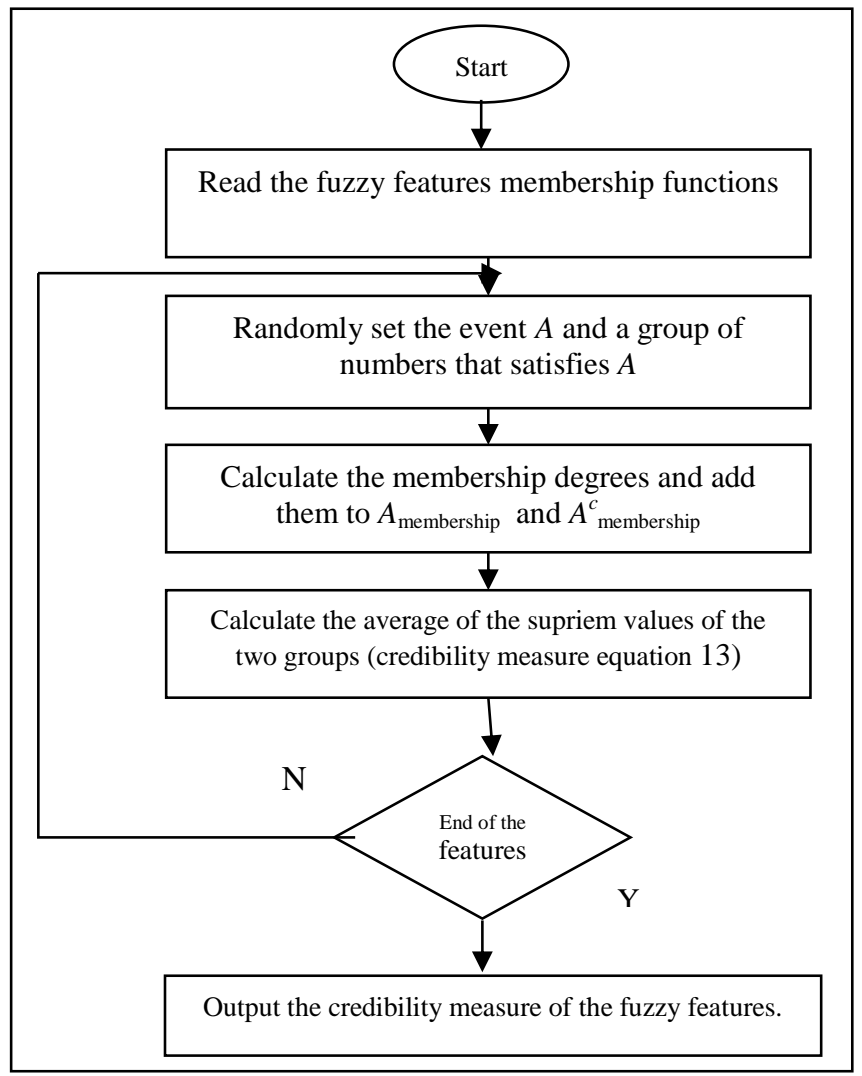

Fig. 4. The Ranking Fuzzy Variables process basing on credibility measure

The number $r$ belongs to the subset Normal. To measure the credibility of the $M C V$ fuzzy variable for the event $A$ as $\xi \in$ Normal, take a set of random value that satisfies the event $A$ like $M c v=82$ that has the membership degrees(Low:0.1, Normal:0.9). The procedure adds the membership degree 0.9 to $\mathrm{A}_{\text {membership }}$ and the membership degree 0.1 to the $\mathrm{A}^{\mathrm{c}}$ membership. Then take another random value like $M C V=99$ with membership degrees (Normal: 0, High: $0.5)$ and add them to the $A_{\text {membership and } A_{\text {membership }}}^{c}$ respectively. Then average of the supreme function of the whole membership degrees in both the event and its complement is calculated to represent the fuzzy variable credibility value. The predefined accepted confidence rate is $70 \%$ 


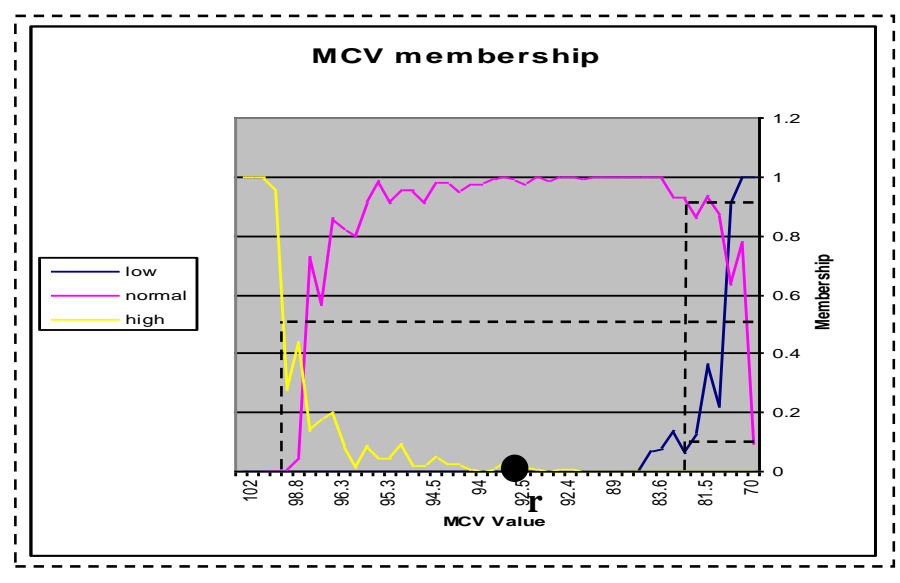

Fig. 5. The fuzzy variable MCV

\section{Generating Fuzzy Rules by ANT Inspired Computation Simultaneous Rule Learning (FRANT IC-SRL)}

FRANT IC-SRL (Fuzzy Rules from ANT-Inspired Computation - Simultaneous Rule Learning)[12] is an Ant inspired optimization algorithm for building fuzzy rules in a simultaneous methodology. The simultaneous technique tends to test the whole rule base induced by the Ants agents instead of testing each rule iteratively. The algorithm initializes the node graph and the pheromone level as the inverse of the node number. Each node is a term that may be added to the constructed rule. The pheromone level is relevant to the node itself instead of the graph edge because the arrangement of nodes inside the rule is not important. Each ant start at a random node then checks adding this node to the rule or not by the minimum number of instances covered. After adding the node the ant goes to the next node with the highest transition rule.

$$
p_{j}^{m}(t)=\frac{\left[\eta_{j}\right] \times\left[T_{j}(t)\right]}{\sum_{i \in I_{m}}\left[\eta_{i}\right] \times\left[T_{i}(t)\right]}
$$

Where $I_{\mathrm{m}}$ is the number of nodes (terms) that still may be included in the rule antecedent by ant $m$ and $j$ is the nodes in the node graph. If one term of a fuzzy variable is added to the rule antecedent the $I_{\mathrm{m}}$ will exclude the other linguistic values of those fuzzy variables. This constraint prevents the rule antecedent from containing conflict propositions. $\eta_{j}$ is the node heuristic value and $T_{j}(t)$ is the node pheromone level. The transition rule is probabilistic but biased to the node pheromone level and heuristic value.

The pheromone level is a guide for the ant. This guide is a clue of the importance level of a node (demonstrated by the ants visited the node before). The pheromone level is updated by the algorithm using the best rule base nodes.

$$
T_{j}(t+1)=T_{j}(t)+\left(T_{j}(t) * Q\right)
$$

Where $j$ is the number of nodes in best rule base, $t$ is the time step and $Q$ is the quality of rule induced by the accuracy level of correct instances classified by the rule.

The heuristic value describes the association between a term (node) $j$ and the class. If a term is has a heuristic value for a certain class then the ACO will use this term in rules leading to that class.

$$
\eta_{j}=\frac{\sum_{u \in U} \min \left(\mu_{A}(u), \mu_{B}(u)\right)}{\sum_{u \in U} \mu_{A}(u)}
$$

Where $u$ is an instance in the universe of discourse $U, A$ represents a class label and $B$ represent a term that may be added to the rule antecedent. The algorithm pseudo-code is illustrated in Figure 6.

\section{Credibility Measure for the System}

The system equation is the (if-then) rules set resulted from the FRANT IC-SRL Algorithm Process. Applying unseen instances to these rules gives the result of the whole system. The number of correct instances classified is the accuracy rate of this system. The credibility of the system depends on the confidence level given to the result of the (if-then) rules set. Hence, measuring the credibility value for the result of unseen instances (class attributes) demonstrate the credibility level of the system.

Equation 13 calculates the credibility value for the fuzzy attribute according to a random event $A$. The class attribute values are derived by testing the system by the unseen instances. Hence, applying the equation 13 on the class attributes calculates the credibility value of the system. 


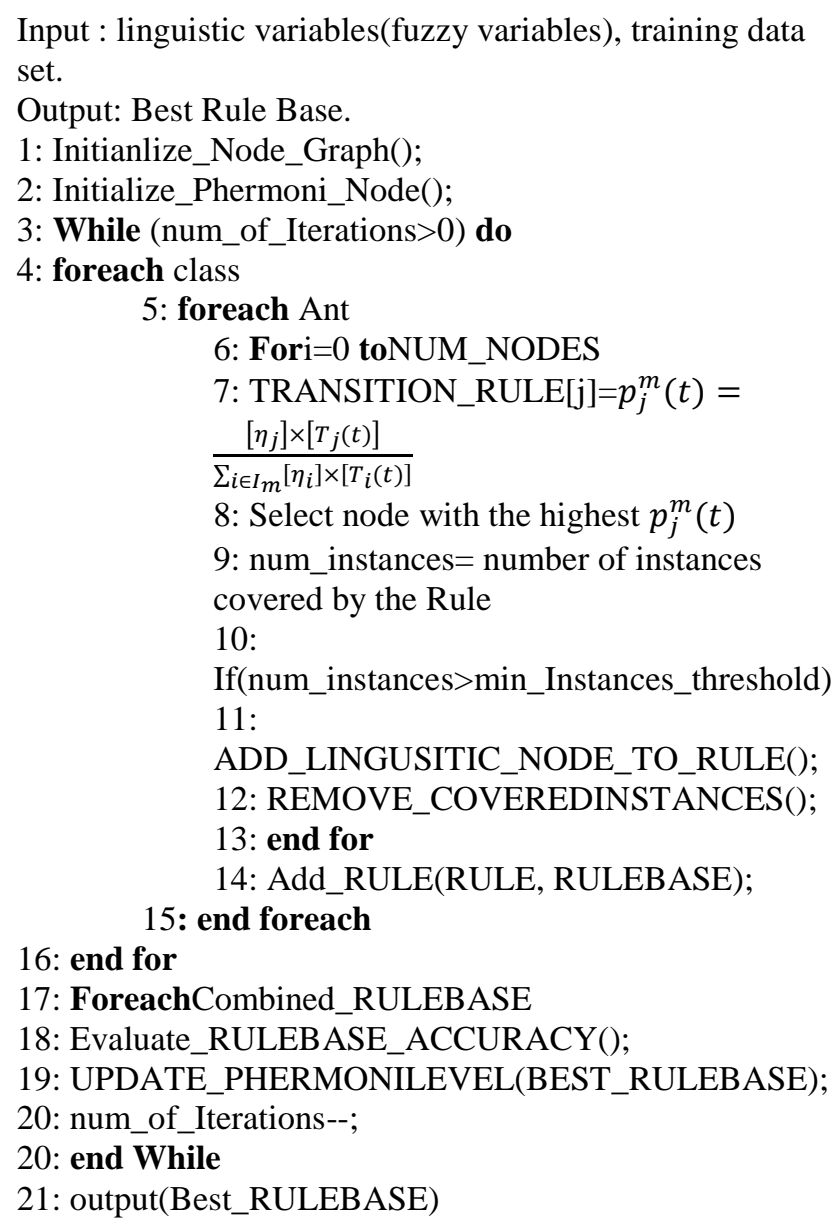

Fig. 6. The FRANT IC-SRL algorithm

\section{EXPERIMENTAL RESULTS}

The proposed hybrid model is composed of four main sub models. The first sub model is the Generating fuzzy membership functions for features subsets which is responsible for generating the degree of membership of the values of the variables in their corresponding subsets. This process uses the SOFM, which uses its unsupervised learning and clustering ability to learn the weights of the neural net from the input training data vectors, to obtain the variables values and their corresponding membership degrees. Using these values we can draw an analogue membership function for each subset of the variables. This technique is implemented before in a previous work[4]. The second sub module calculates the credibility value for the generated fuzzy variables using the Credibility Inversion Theorem to rank the confidence level of these variables by the credibility measure. The credible ranked fuzzy attributes are used as the base of the fuzzy rule based system. The third sub model is the generating fuzzy rules module. It applies simultaneous learning capabilities of the FRANT IC-SRL using the Ant Colony Optimization to find the best rule base. The whole system is ranked by the credibility measure of its output classes attribute. The classes attribute values is obtained by applying new instances to the system and take the output and the corresponding membership degree.

The data sets used in this research to test the model are taken from the UCI machine learning repository[6] and their properties are illustrated in Table 1. The data set records are divided in two equal parts (one for the training data and one for the test data).

TABLE I. DESCRIPTION OF THE DATA SETS PROPERTIES

\begin{tabular}{|c|c|c|c|c|c|}
\hline $\begin{array}{c}\text { Name } \\
\text { of the } \\
\text { data } \\
\text { set }\end{array}$ & $\begin{array}{c}\text { No of } \\
\text { attributes }\end{array}$ & $\begin{array}{c}\text { No of } \\
\text { continuous } \\
\text { attributes }\end{array}$ & $\begin{array}{c}\text { No of } \\
\text { categorical } \\
\text { attributes }\end{array}$ & $\begin{array}{c}\text { No of } \\
\text { data } \\
\text { records }\end{array}$ & $\begin{array}{c}\text { No of } \\
\text { classes }\end{array}$ \\
\hline $\begin{array}{c}\text { Breast } \\
\text { Cancer }\end{array}$ & 10 & 10 & 0 & 699 & 2 \\
\hline Liver & 6 & 6 & 0 & 345 & 2 \\
\hline
\end{tabular}

In experiments, the SOFM is trained with 3 or 4 input neurons (one for the feature value and the rest for the subsets) depending on the number of subsets of the features and 15 output neurons that produced 225 of features values and their corresponding membership degrees. These values were used to draw an analogue function for each feature. Based on the SOM associated with the credibility measurethe membership functions of the 6 conditional features of the liver data setare determined, declared by Figure 7. The data liver attributes are recognized by mean corpuscular volume, alkaline phosphates, alamine aminotransferase, aspirate aminotransferase, gammaglutamyltranspeptidase and number of half-pint equivalents of alcoholic. The $7^{\text {th }}$ attribute is a class selector field used to split the data into two sets. 


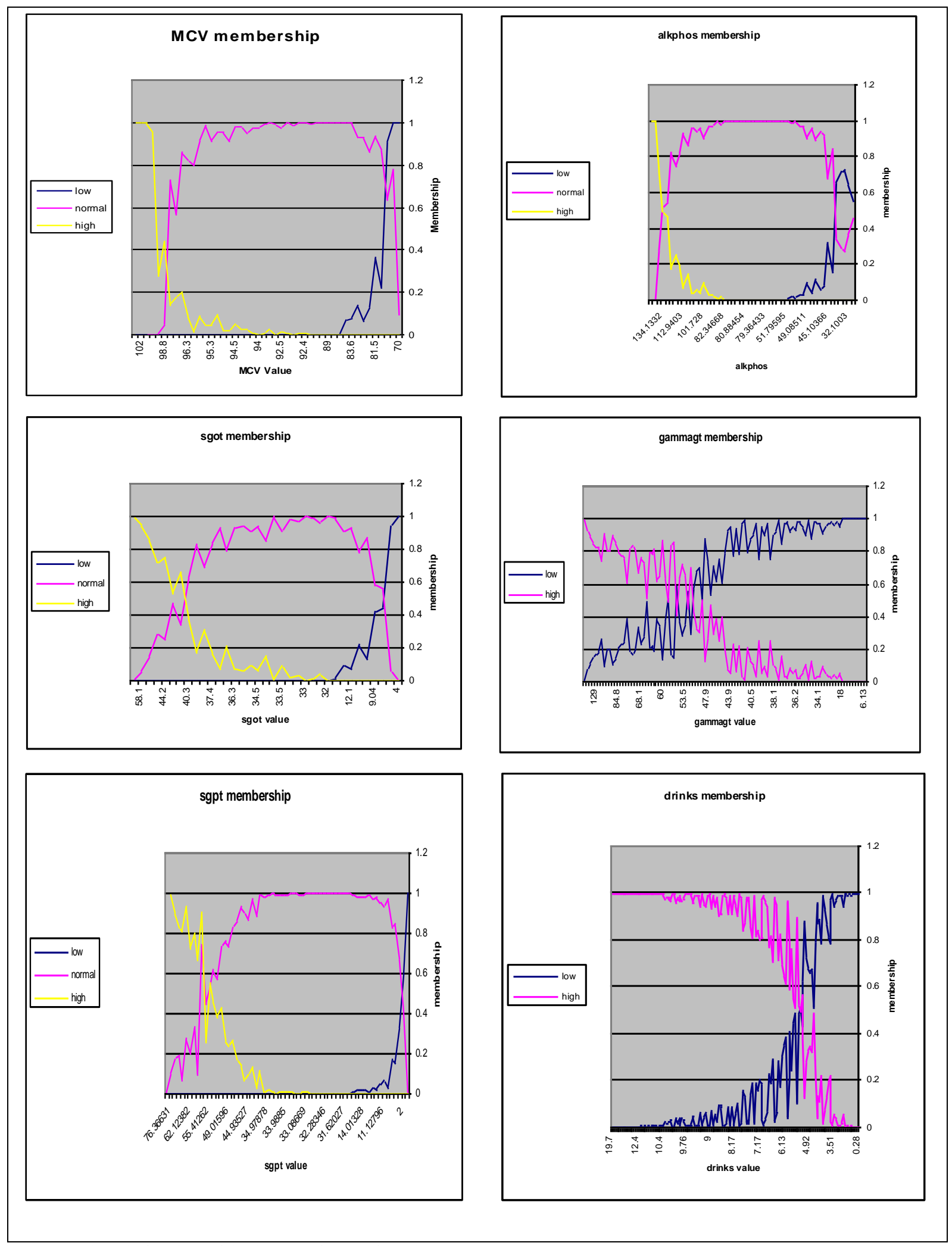

Fig. 7. Membership functions of the liver data features from the SOM process

The credibility measure is used to calculate the weighted average of the confidence level of the generated fuzzy attributes. The features membership functions are passed to the credibility measure procedure. The inverse credibility equation is used to calculate the credibility of a random event assumed on each feature. A sufficiently large set of fuzzy numbers is created randomly in the event data space and the membership degree of these numbers are taken in both the 
event $A_{\text {membership }}$ and the event complement $A^{\mathrm{c}}$ membership. The credibility values calculated for the fuzzy variables in the liver disorder data set are presented in Table 2.

The accepted credible fuzzy variables should have a credibility value that exceeds a predefined threshold. In experiment, the predefined confidence level threshold is $70 \%$. The fuzzy variable that exceeds that threshold will continue in the fuzzy rough rule based system development.

TABLE II. FUZZY VARIABLES CREDIBILITY VALUES

\begin{tabular}{|l|l|}
\hline \multicolumn{1}{|c|}{ Fuzzy variable name } & Credibility value \\
\hline mcv & 0.95 \\
\hline Alkphos & 0.88 \\
\hline Sgpt & 1 \\
\hline Sgot & 0.97 \\
\hline Gammagt & 0.65 \\
\hline Drinks & 1 \\
\hline
\end{tabular}

The most credible fuzzy variables are listed in the Table 3. The most credible fuzzy variables are the input of the fuzzy rough reduction procedure.

TABLE III. MOST CREDIBLE FUZZY VARIABLES

\begin{tabular}{|l|l|}
\hline Fuzzy variable name & Credibility value \\
\hline mcv & 0.95 \\
\hline Alkphos & 0.88 \\
\hline Sgpt & 1 \\
\hline Sgot & 0.97 \\
\hline Drinks & 1 \\
\hline
\end{tabular}

The FRANT IC-SRL algorithm initializes a node graph for the Ants agents to run. The nodes are the conditional terms of the rule antecedent. The ant starts randomly at any node then tracks the nodes with the highest pheromone level. After the term is added to the rule the instances covered by that term is removed from the training data and the variable of that term is removed from the list of nodes to be visited next. After all the ants find their rule bases, the best rule base is used to update the pheromone level of the node graph. At the end of iterations the best rule based is output as the fuzzy classification final rule base.

The credibility value of the class attribute demonstrates the confidence level of fuzzy rule based system. This process applies Equation 16 to the class attributes values and calculates the credibility value. The credibility of the liver disorder data set on the system is $59 \%$ and the Breast Cancer data set is $88 \%$. The average credibility level of the system for both the data sets is $73 \%$. The comparison between the proposed model and other techniques is listed in Table 4. The resultsproved the accuracy levels of the rule sets generated by C4.5, Naïve Bays, SOFM \& PGA[13] are less classified than the proposed model (SOFM + FRANT IC-SRL using Credibility Measure) applied on two different data sets (liver and breast cancer data sets)as illustrated in Figure 8 .

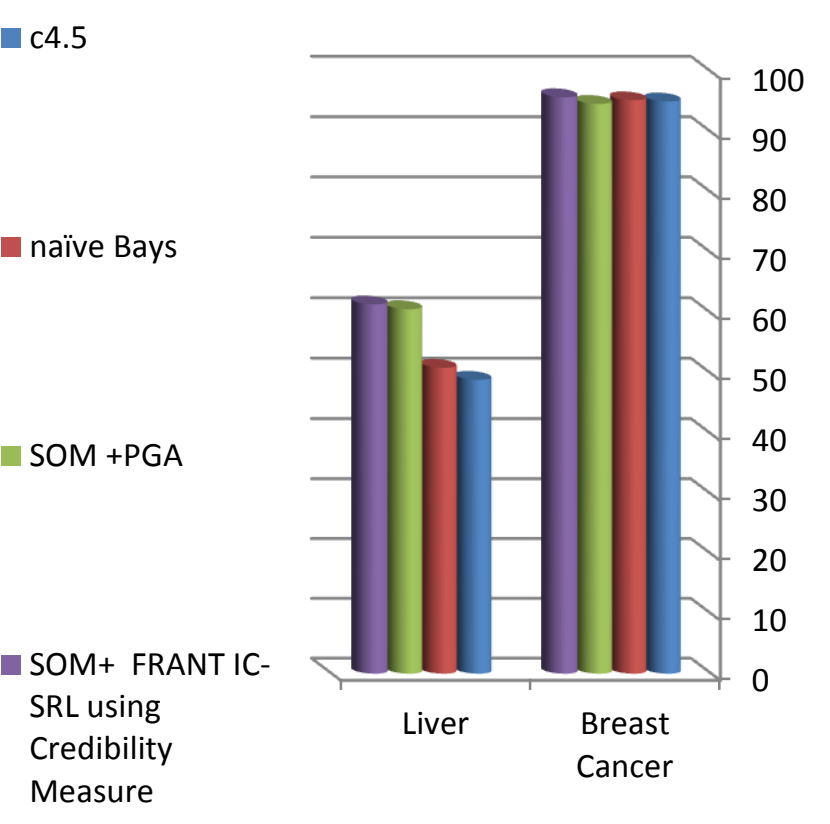

Fig. 8. the accuracy of the rule set of the proposed model and some other rule generator algorithms

TABLE IV. COMPARISON BETWEen THE PROPOSED MOdEL AND OTHER TECHNiQues Found IN THE FIELD OF GENERATING FuZZY Rules

\begin{tabular}{|c|c|c|c|c|}
\hline & c4.5 & $\begin{array}{c}\text { naïve } \\
\text { Bays }\end{array}$ & $\begin{array}{c}\text { SOM } \\
\text { +PGA }\end{array}$ & $\begin{array}{c}\text { SOM+ FRANT IC- } \\
\text { SRL using Credibility } \\
\text { Measure }\end{array}$ \\
\hline $\begin{array}{c}\text { Breast } \\
\text { Cancer }\end{array}$ & 95.1 & 95.3 & 94.7 & 95.8 \\
\hline Liver & 49 & 51 & 60.7 & 61.5 \\
\hline
\end{tabular}

These comparisons show that the proposed hybrid model gave better accuracy level than the previous ones. The results indicate an average accuracy of around $78.8 \%$. This compares favorably with previous systems for classifying documents, whose average accuracy is $74.3 \%$.

\section{CONCLUSIONS}

Fuzzy classification Rules are a reliable way to handle uncertainty in real world sincethe clustering phenomenon of defects becomes significant task. Fuzzy clustering depends totally on fuzzy propositions which in turn use fuzzy variables instead of regular ones. In designing more significant fuzzy clustering based on fuzzy rules, there are four restrictions that prevent any heuristic model to achieve more accurate results.

First, preparing a correspondence membership function that maps each fuzzy variable. Second, ranking the generated fuzzy variables wherever the lack of self-dual problem should be solved. Third, generating fuzzy dynamic (if-then) rules structure, that efficiently represents the concludeduncertain knowledge. Finally, measuring the credibility of the whole fuzzy system to be compared among different systems. 
This paper introduces a hybrid model based on the metaheuristic algorithms. A novel clustering approach that improves the clustering task for uncertain knowledge is introduced, where the four restrictions are handled. Clustering based on fuzzy relationship communicate efficiently with the predefined problems and achieve more flexible and dynamic rules.

This paperproved the ability of SOFM to learn and cluster its inputs can help in clustering fuzzy variable into its corresponding fuzzy subsets and finding the representative fuzzy membership functions of the input subsets. By SOFM, a fuzzy clustering is first generated which suffer from the lake of self-dual problem. The credibility measure is used to calculate the weighted average of the fuzzy variable confidence level using their membership functions.The Credibility Inversion Theorem solve the ranking problem. For designing dynamical fuzzy rules, a meta-heuristic search strategy is needed. The FRANT IC-SRL algorithm uses the Ant Colony Optimization capabilities to find the best fuzzy rules depending on its simultaneous technique. The algorithm initializes the node graph. Each node represents a term that could be added to the rule antecedent. The ants follow the pheromone level updated by the best rule base induced during iterations. The resulting fuzzy rule base is further tested by means of testing data set to make sure that the accuracy meets the effective levels of the performance. The whole system is ranked by the credibility value of its classes attribute. The experiment results are shown and they proved that the proposed hybrid model is much more efficient than previous techniques.

Although the hybrid model of SOFM and FRANT IC-SRL algorithm achieves some contribution in improving classification accuracy, it is still not able to handle the missing attribute value. Though, some generalization in preparing the initial local rules is needed. The generalization of tolerance rough approximations to fuzzy environments should play an important role in the development of uncertain knowledge. A modified similarity relation is defined on the predefined fuzzy concepts to yield an approximation space of lower and upper approximations. The generalized discernibility matrix reduces the fuzzy superfluous variables. Thus, the credibility inversion theorem can be used in advance to rank the most significance fuzzy variables. In the post processing, a suggested fuzzy cellular automata model is defined as an emergent system to conclude more robust dynamical rules. The new suggested model are hoped to achieve more accurate results.

\section{REFERENCES}

[1] Alex A. Freitas, "Evolutionary Algorithms for Data Mining" , Data Mining and Knowledge Discovery Handbook, ISBN: 978-0-387-244358, Springr-Verlag, New York, USA, pp. 435-467, 2005.

[2] Bilal Alatas and Erhan Akin, " FCACO: Fuzzy Classification Rules Mining Algorithm with Ant Colony Optimization", Advances in Natural Computation, ISBN 978-3-540-28320-1, Online ISBN 978-3-54031863-7, Springer Berlin Heidelberg, Vol. 3612, pp 787-797, 2005.

[3] Bo Liu, Hussein A. Abbass and Bob McKay, "Classification Rule Discovery with Ant Colony Optimization", IEEE Computational Intelligence Bulletin, Vol.3 No.1, pp. 31-35, 2004.

[4] Chih-Chung Yang, N.K. Bose, "Generating fuzzy membership function with self-organizing feature map", Pattern Recognition Letters, Elsevier Science Inc. New York, NY, USA, Vol. 27, No. 5, pp. 356-365, 2006
[5] Fernando E. B. Otero, Alex A. Freitas, Colin G. Johnson, " cAnt-Miner: An Ant Colony Classification Algorithm to Cope with Continuous Attributes", Ant Colony Optimization and Swarm Intelligence, Print ISBN 978-3-540-87526-0, Online ISBN 978-3-540-87527-7, Vol. 5217, pp 48-59, 2008.

[6] K. Bache and M. Lichman, "UCI Machine Learning Repository [http://archive.ics.uci.edu/ml], Irvine, CA: University of California, School of Information and Computer Science, 2013.

[7] Lotfi A. Zadeh, "From Computing with Numbers to Computing with Words - from Manipulation of Measurements to Manipulation of Perceptions", in International Journal of Applied Math and Computer Science, Vol. 12, No. 3, pp. 307-324, 2002.

[8] Liu B, "A survey of credibility theory", Fuzzy Optimization and Decision Making, Vol.5 No. 4, pp. 387-408, 2006.

[9] Liu B," A Survey of Entropy of Fuzzy Variables", Journal of Uncertain Systems, Vol.1 No. 1, pp.4-13, 2007.

[10] Liu B, and Liu YK, "Expected value of fuzzy variable and fuzzy expected value models", IEEE Transactions on Fuzzy Systems, Vol.10 No. 4, pp.445-450, 2002.

[11] Marie-Claire Koissi and Arnold F. Shapiro," Credibility Theory in a Fuzzy Environment", The 47th Actuarial Research conference Proc., University of Manitoba, Canada, 2012.

[12] Michelle Galea and Qiang Shen, "Simultaneous Ant Colony Optimization Algorithms for Learning Linguistic Fuzzy Rules", Swarm Intelligence in Data Mining, Print ISBN 978-3-540-34955-6, Online ISBN 978-3-540-34956-3, Springer Berlin Heidelberg, Vol. 34, pp 7599, 2006.

[13] Mona Gamal, Ahmed Abou El-Fetouh, ShereefBarakat and ElsayedRadwan, "A Hybrid of Self Organized Feature Maps and Parallel Genetic Algorithms for Uncertain Knowledge", International Journal of Computer Applications, Vol. 60, No.6, pp.23 - 31, 2012.

[14] Qifang, Tiezhu Wang, Jingyao Zhu and Zutong Wang," A Hybrid Intelligent Algorithm for Fuzzy Programming Problem under Credibility Theory", Applied Mechanics and Materials, Vol. 530-531, pp 363-366, 2014

[15] T. Kohonen, "Self-Organizing Maps", Springer Series in Information Sciences, Vol. 30, ISBN 3-540-67921-9, ISSN 0720-678X, Springer Berlin Heidelberg, New York, 2001.

[16] RituparnaChutia, SupahiMahanta and D. Datta, "Arithmetic of Triangular Fuzzy Variable from Credibility Theory", International Journal of Energy, Information and Communications, Vol. 2, No. 3, pp. 9-202011.

[17] Sanjeev Gupta and Sanjeev Bhardwaj," Rule Discovery for Binary Classification Problem using ACO based Antminer", International Journal of Computer Applications, Volume 74, No. 7, pp. 19-23, 2013.

[18] Urszula Markowska-Kaczmar and Wojciech Trelak, "Extraction of fuzzy rules from trained neural network using evolutionary algorithm ", ESANN 11th European Symposium on Artificial Neural Networks Proc., Bruges, Belgium, ISBN 2-930307-03-X, pp. 149-154, 2003.

[19] V.S.Vaidyanathan, "Wilcoxon signed rank test for imprecise observations", IOSR Journal of Mathematics (IOSR-JM), Vol. 10, No. 2, pp. 55-59, 2014.

[20] Vishal Arora, Vadlamani Ravi, " Data Mining using Advanced Ant Colony Optimization Algorithm and Application to Bankruptcy Prediction", International Journal of Information Systems and Social Change archive, IGI Publishing Hershey, PA, USA, Vol 4, No. 3, pp. 33-56, 2013.

[21] Xiang Li and Baoding Liu, " A Sufficient and Necessary Condition for Credibility Measures", International Journal of Uncertainty, Fuzziness and Knowledge-Based Systems, Vol.14, No.5, pp. 527-535, 2006.

[22] Xiaxia Huang, "Chance-constrained programming models for capital budgeting with NPV as fuzzy parameters", Journal of Computational and Applied Mathematics, Vol. 198, No.1, pp. 149 - 159, 2007.

[23] Zhi-qiang Liu and Zhi-Qiang Liu "Fuzzy Possibility Space and Type-2 Fuzzy Variable", Foundations of Computational Intelligence, 2007. FOCI 2007. IEEE Symposium on , Print ISBN: 1-4244-0703-6, pp. 616$621,2007$. 